\title{
Relatório de pareceristas da Revista de Sociologia e Política em 2008
}

Board of Reviewers of Revista de Sociologia e Política in 2008

\section{Relación de evaluadores de la Revista de Sociologia e Política en 2008}

Adolpho Carlos Françoso Queiróz (Universidade Metodista) ad hoc

Adriana Aparecida Marques (USP) ad hoc

Adriana Cristina Omena dos Santos (UFU) ad hoc

Adriano Codato (UFPR)

Adriano de Freixo (Cefet-RJ) ad hoc

Adriano Oliveira dos Santos (UFPE) ad hoc

Aldomar Arnaldo Rückert (UFRGS) ad hoc

Alexandre Fortes (UFRRJ) ad hoc

Álvaro Gabriel Bianchi Mendez (Unicamp)

Alzira Alves de Abreu (FGV) ad hoc

Ana Cláudia Duarte Rocha Marques (USP) ad hoc

Ana Cleide Chiarotti Cesário (UEL) ad hoc

Ana Lúcia Pastore Schritzmeyer (USP) ad hoc

Ana Luisa Fayet Sallas (UFPR) ad hoc

Ana Paula Tostes (USP) ad hoc

André de Faria Pereira Neto (Fiocruz) ad hoc

André Filipe Pereira Reid dos Santos (Faculdade Estadual de Ciências e Letras de Campo Mourão) ad hoc

André Luiz Marenco dos Santos (UFRGS) ad hoc

André Moysés Gaio (UFJF) ad hoc

Andréia Galvão (Unicamp) ad hoc

Antônio Carlos Rafael Barbosa (UFF) ad hoc

Antônio Luigi Negro (UFBA) ad hoc

Arilson da Silva Favareto (UFABC) ad hoc

Armando Boito Júnior (Unicamp)

Basilia Aguirre (USP) ad hoc

Breno Augusto Souto Maior Fontes (Universidade de Coimbra) ad hoc

Bruno Sciberras de Carvalho (UFRJ) ad hoc

Carla Gonçalves Antunha Barbosa (INEP) ad hoc

Carlos Augusto Teixeira Magalhães (Centro Universitário Newton Paiva) ad hoc

Carlos R. S. Milani (UFBA) ad hoc

Carlos Ranulfo de Melo (UFMG) ad hoc

Carlos Sell (UFSC) ad hoc

Cécile Hélène Jeanne Raud (UFSC) ad hoc

Celso Castro (FGV)

Ciméa Barbato Bevilaqua (UFPR) ad hoc

Cláudia Feres Faria (UFMG) ad hoc

Cláudio Chaves Beato Filho (UFMG) ad hoc

Cláudio Henrique de Moraes Batalha (Unicamp) ad hoc

Corinne Davis Rodrigues (UFMG) ad hoc

Cristiane Kerches da Silva Leite (USP) ad hoc

Cristiano Fonseca Monteiro (UFF) ad hoc

Delmo Mattos da Silva (FGV) ad hoc

Édison Luís Gastaldo (Unisinos) ad hoc

Eduardo Antônio Salomão Condé (UFJF) ad hoc

Eduardo Lopes Cabral Maia (UFSC) ad hoc

Eduardo Rozenthal (USU) ad hoc

Elizabeth Cancelli (USP) ad hoc 
Elsio Lenardão (UEL) ad hoc

Emerson Urizzi Cervi (UFPR)

Estevão Chaves de Rezende Martins (UnB) ad hoc

Eugênio Carlos Ferreira Braga (Unicamp) ad hoc

Fabiano Engelman (Ufscar) ad hoc

Fabiano Santos (Iuperj) ad hoc

Fabrício Ricardo de Limas Tomio (UFPR)

Fernando Coutinho Cotanda (UFRGS) ad hoc

Fernando Lattman-Weltman (FGV) ad hoc

Flávio Ramos (Univali) ad hoc

Francisca Verginio Soares (Observatório Social Londrinense) ad hoc

Giuseppe Mario Cocco (UFRJ) ad hoc

Giuseppina Rosaria de Grazia (Universidade de Santo Amaro) ad hoc

Gláucia Angélica Campregher (Unisinos) ad hoc

Gustavo Biscaia de Lacerda (UFPR)

Hélio Rebello Cardoso Júnior (Unesp) ad hoc

Hélio Ricardo do Couto Alves (FURG) ad hoc

Heloiza Matos (Faculdade Cásper Líbero) ad hoc

Henrique Caetano Nardi (UFRGS) ad hoc

Honor de Almeida Neto (Ulbra) ad hoc

Hugo Alberto Borsani Cardozo (UENF) ad hoc

Ilza Araújo Leão de Andrade (UFRN) ad hoc

Ingrid Piera Andersen Sarti (UFRJ) ad hoc

Ivann Carlos Lago (Univali) ad hoc

Jadir Antunes (Unioeste) ad hoc

João Trajano Sento-Sé (UERJ) ad hoc

Jorge Zaverucha (UFPE) ad hoc

José Carlos Reis (UFMG) ad hoc

José Luís Bizelli (Unesp) ad hoc

José Luiz Ratton (UFPE) ad hoc

Josué Pereira da Silva (Unicamp) ad hoc

Juliana Lyra Viggiano Barroso (USP) ad hoc

Kai Michael Kenkel (PUC-RJ) ad hoc

Klaus Frey (PUC-PR) ad hoc

Laurindo Mékie Pereira (Unimontes) ad hoc

Leila Borges Dias Santos (UEG) ad hoc

Leila Stein (Unesp) ad hoc

Leonardo Barbosa e Silva (UFU) ad hoc

Letícia Maria Schabbach (Universidade de Sta. Cruz do Sul) ad hoc

Lígia Luchmann (UFSC) ad hoc

Loreley Gomes Garcia (UFPB) ad hoc

Luciane Cristina de Oliveira (Unesp) ad hoc

Luciano Cavini Martorano (Iuperj) ad hoc

Luís Antônio Francisco de Souza (Unesp) ad hoc

Luís Felipe Miguel (UnB)

Luís Fernando Ayerbe (Unesp) ad hoc

Luís Gustavo Mello Grohmann (UFSM) ad hoc

Luiz Moraes de Niemeyer Neto (PUC-SP) ad hoc

Marcello Simão Branco (USP) ad hoc

Marcelo de Almeida Medeiros (UFPE) ad hoc

Marcia da Silva Mazzon (UFSC) ad hoc

Márcia Maria Menendes Motta (UFF) ad hoc

Marco Antônio Perruso (UFRJ) ad hoc

Marcos Alexandre dos Santos Ferraz (USP) ad hoc

Marcos Cassin (USP) ad hoc 
Marcos Del Roio (Unesp) ad hoc

Margaret Marchiori Bakos (PUC-RS) ad hoc

Maria Alice Rosa Ribeiro (Unesp) ad hoc

Maria Arminda do Nascimento Arruda (USP)

Maria Beatriz Bianchini Bilac (Unimep) ad hoc

Maria de Fátima Franco dos Santos (Puccamp) ad hoc

Maria de Fátima Piazza (UFSC) ad hoc

Maria do Socorro Sousa Braga (Ufscar) ad hoc

Maria Eliana Labra (Fiocruz) ad hoc

Maria Lígia de Oliveira Barbosa (UFRJ) ad hoc

Maria Salete Souza de Amorim (Unioeste) ad hoc

Maria Victória Espiñeira Gonzalez (UFBA) ad hoc

Mário Fuks (UFMG)

Míriam de Oliveira Santos (UFRRJ) ad hoc

Nelson Rosário de Souza (UFPR)

Neusa Maria Sens Bloemer (Univali) ad hoc

Nildo Silva Viana (UEG) ad hoc

Paolo Ricci (USP) ad hoc

Paulo José dos Reis Pereira (PUC-SP) ad hoc

Paulo Vizentini (UFRGS) ad hoc

Raul Francisco Magalhães (UFJF) ad hoc

Regina Beatriz Guimarães Neto (UFMT) ad hoc

Renarde Freire Nobre (UFMG) ad hoc

Renato Luís do Couto Neto e Lemos (UFF) ad hoc

Renato Monseff Perissinotto (UFPR)

Renato Sérgio de Lima (Fundação Seade) ad hoc

Roberto Mauro Cortez Motta (UFPB) ad hoc

Roberto Véras de Oliveira (UFCG) ad hoc

Ronaldo de Noronha (UFMG) ad hoc

Rosimary Gonçalves de Souza (UERJ) ad hoc

Rossana Rocha Reis (USP) ad hoc

Ruthy Nadia Laniado (UFBA) ad hoc

Sadi Dal Rosso (UnB) ad hoc

Samantha Viz Quadrat (UFF) ad hoc

Sandra Cristina Gomes (Cebrap) ad hoc

Scott William Hoefle (UFRJ) ad hoc

Sérgio Soares Braga (UFPR)

Sérgio Tiski (UEL) ad hoc

Sílvio José Benelli (Unesp) ad hoc

Simone Rodrigues Bohn (Universidade de York, Canadá) ad hoc

Tade-Ane Amorim (Unisul) ad hoc

Tamara Benakouche (UFSC) ad hoc

Tomas Antônio Moreira (PUC-PR) ad hoc

Vanessa Elias de Oliveira (PUC-SP) ad hoc

Yan de Souza Carreirão (UFSC) ad hoc 